\title{
Médiévales
}

Langues, Textes, Histoire

46 | printemps 2004

Éthique et pratiques médicales

\section{«Le roi refuse l'or de ses sujets ». Analyse d'une miniature du «Livre de bonnes meurs » de Jacques Legrand ( $†$ 1415)}

"The King refuses the gold of his subjects ». Study of a miniature of Jacques

Legrand's Livre de bonnes meurs (ca 1410).

\section{Lydwine Scordia}

\section{OpenEdition \\ Journals}

Édition électronique

URL : https://journals.openedition.org/medievales/1049

DOI : 10.4000/medievales. 1049

ISSN : $1777-5892$

\section{Éditeur}

Presses universitaires de Vincennes

\section{Édition imprimée}

Date de publication : 1 juin 2004

Pagination : 109-130

ISBN : 2-84292-151-8

ISSN : 0751-2708

\section{Référence électronique}

Lydwine Scordia, « «Le roi refuse l'or de ses sujets ». Analyse d'une miniature du « Livre de bonnes meurs » de Jacques Legrand († 1415) », Médiévales [En ligne], 46 I printemps 2004, mis en ligne le 02 mars 2006, consulté le 22 avril 2022. URL : http://journals.openedition.org/medievales/1049 ; DOI : https://doi.org/10.4000/medievales. 1049

Ce document a été généré automatiquement le 22 avril 2022.

Tous droits réservés 


\title{
«Le roi refuse l'or de ses sujets».
} Analyse d'une miniature du « Livre de bonnes meurs » de Jacques Legrand $(† 1415)$

\author{
"The King refuses the gold of his subjects ». Study of a miniature of Jacques \\ Legrand's Livre de bonnes meurs (ca 1410).
}

\section{Lydwine Scordia}

1 En 1410, l'augustin Jacques Legrand (ca 1365-1415) offre son Livre de bonnes meurs au duc Jean de Berry ${ }^{1}$. Dans l'une des copies du manuscrit, datant des années 1490, se trouve la belle miniature qui sert de point de départ à cette recherche. On y voit des sujets qui tendent au roi des plats remplis de pièces d'or ${ }^{2}$. Le cadre historique de la rédaction et de la décoration de ce traité couvre donc tout le $\mathrm{xv}^{\mathrm{e}}$ siècle ${ }^{3}$. Au début du siècle, l'impôt direct n'est ni régulier ni permanent ${ }^{4}$. La réflexion sur la légitimité du prélèvement fiscal est pourtant déjà ancienne. La question d'un impôt levé par le roi sur l'ensemble des sujets est d'abord posée sous le règne de Philippe IV, dans un contexte de forte croissance des dépenses militaires. Un demi-siècle plus tard, le paiement de la lourde rançon du roi Jean II, fait prisonnier à Poitiers, régularise la perception pendant vingt ans. Quelle que soit la cause - militaire le plus souvent -, le prélèvement royal est étroitement lié à une finalité précise. Et « lorsque la cause cesse, l'effet cesse $»^{5}$. Il faut attendre 1445 pour que le roi Charles VII pérennise la taille. L'armée est alors devenue permanente, et avec elle, le moyen de la financer. Sous le règne de Louis XI, la taille est multipliée par trois. À la mort du roi, les États généraux de Tours réagissent et rappellent que les revenus du domaine restent le fondement financier des dépenses; les États accepteront ponctuellement d'accorder les sommes nécessaires au prince par « don et ottroy », et non autrement ${ }^{6}$.

2 Alors que les rois disposent depuis la fin du xiiie siècle d'une argumentation intellectuelle légitimant l'impôt, la théorie domaniale persiste ${ }^{7}$. Chacun continue de penser que le roi doit « vivre du sien », c'est-à-dire qu'il doit se contenter des revenus 
de son domaine. Le roi lui-même ne cesse de s'excuser du prélèvement fiscal dont il promet qu'il sera de courte durée. Or les dépenses ont augmenté, alors que les revenus ordinaires se sont effondrés et ne représentent plus que $50 \%$ des recettes en 1330, $3 \%$ sous Charles VII ${ }^{8}$. La situation est matériellement difficile pour un prince écartelé entre les réalités du temps et l'idéal d'une royauté ministérielle.

Les représentations du prince levant l'impôt brillent donc par leur rareté. Le prince nourrit les pauvres, il ne prélève pas d'argent. Il peut être figuré comme le garant de l'équité des comptes du royaume, mais il ne touche pas matériellement les pièces d'or' . Il est le roi-berger qui garde son troupeau et ne tond ses brebis qu'une fois l'an, « sans du cuir écorcher $»^{10}$. En revanche, les artistes stigmatisent l'avarice royale. L'une des images les plus intéressantes n'est-elle pas celle qui place un roi couronné dans le chaudron d'enfer, une bourse symbolisant son crime attachée au cou ${ }^{11}$ ?

Ce préambule explique la prudente curiosité qui saisit l'historien en regard de cette miniature extraite du Livre de bonnes meurs. Elle nous conduira à commencer par une description et une analyse de l'image, puis à poser la question de la récompense terrestre du prince, et enfin à aborder le thème apparemment anachronique du «salaire du roi ».

«Le roi refuse l'or de ses sujets »

Jacques Legrand, Le Livre de bonnes meurs

(ms. Chantilly, Condé, 297, fo $79 v^{\circ}$, droits réservés)

(Cette image est présente dans la version imprimée du numéro 46)

Un roi, de l'or et des sujets : la miniature représente-t-ellele roi levant l'impôt ?

5 Le duc d'Aumale (1822-1897), cinquième fils de Louis-Philippe, rassembla à Chantilly une très belle collection de manuscrits et livres rares. Considéré comme l'un des plus grands bibliophiles de son temps, c'est lui qui acheta en 1856 le manuscrit des Très Riches Heures du duc de Berry. On trouve aussi, parmi les centaines de pièces de sa collection, « ce beau manuscrit parfaitement conservé, [...] surtout curieux pour l'étude du costume. Il a été acheté pour moi à la vente de M. Boorlut de Noortdonck (Gand, juin 1858) $»^{12}$. Le manuscrit du Livre de bonnes meurs compte effectivement une belle série de miniatures, parmi lesquelles celle que la photothèque Dagli Orti a dotée de la légende suivante : «Un prince reçoit l'impôt or de ses sujets ${ }^{13}$.

Description de la miniature

6 Le manuscrit Condé 297 de Chantilly est un in quarto sur vélin de 225 sur $170 \mathrm{~mm}^{14}$. Il compte 156 folios de 23 lignes par page $\mathrm{e}^{15}$. Les rubriques sont en rouge, les pieds-demouches en bleu. Les initiales ornées accueillent des décors floraux. Le manuscrit a été enluminé dans la vallée de la Loire vers $1490^{16}$. Il compte 53 miniatures, autant que de chapitres ${ }^{17}$. La palette de l'artiste privilégie le rose, le rouge, l'ocre et le bleu. Les scènes extérieures présentent des décors assez similaires, formés de châteaux, de rochers, d'arbres et de différentes sortes de végétaux. Les scènes intérieures sont en revanche extrêmement variées. Ce sont probablement ces dernières qui ont attiré l'attention du duc d'Aumale, car elles offrent une grande diversité vestimentaire des personnages (rois, clercs, étudiants, musiciens ou hommes d'armes) qui incarnent vices et vertus propres à tout traité du bon gouvernement.

7 La miniature du « roi recevant l'impôt » mesure 100 sur $98 \mathrm{~mm}$; elle couvre environ la moitié de la page ${ }^{18}$. L'image déborde de son cadre : les tours du château pointent leurs flèches au-delà du contour doré. L'artiste a représenté un roi, des sujets, et de l'or. Mais fallait-il en conclure que le roi recevait l'impôt de ses sujets? 
8 La miniature rassemble quatre personnages dans une scène d'extérieur ayant pour arrière-plan un château fortifié pourvu de tours, d'un côté, et une campagne hérissée de rochers, de l'autre. Les têtes des quatre hommes arrivent presque au niveau des montagnes et des tours du château. Le personnage principal se trouve à droite sur un plan légèrement surélevé, c'est un roi couronné présenté de face. Il porte un manteau rouge, fourré et bordé d'hermine, par-dessus une robe bleue à manches longues. Ce roi est barbu, il a les sourcils froncés. Sa barbe et ses cheveux blonds lui mangent le visage tant ils sont frisés et volumineux. Le roi porte une couronne haute, en or, posée sur un bonnet rouge. Il a les avant-bras pliés. Ses deux mains sont ouvertes vers l'extérieur. Son regard se porte sur la gauche, en direction des trois personnages qui forment l'autre partie de la miniature.

9 Ces hommes représentés de profil sont vêtus de costumes distincts. Le premier, en cheveux, habillé de chausses noires, porte un habit rouge, court et sans manche, fourré et ourlé d'hermine. Sa chemise est jaune, fermée par des liens aux poignets et à l'encolure; c'est un laïc. Il a les cheveux mi-longs et tient un bonnet dans sa main gauche. Un peu en retrait se trouvent un premier clerc aux cheveux châtain foncé, en longue robe bleue et bonnet carré noir (une barrette), et un second, à barrette et robe rouges, dont on ne distingue bien que la tête. D'un même mouvement, deux d'entre eux tendent au roi des plats remplis de pièces d'or, et le troisième, une bourse de bonne taille.

10 L'attitude du roi symbolise le mécontentement et le refus. Refuse-t-il l'or que viennent lui apporter clercs et laïcs? La lecture du traité de Jacques Legrand permet d'interpréter la scène.

Le roi refuse une récompense financière

11 Cette miniature accompagne Le Livre de bonnes meurs, traité moral dont on connait deux rédactions, l'une en 1404 et l'autre en $1410^{19}$. Le célèbre prédicateur Jacques Legrand a dédié ce texte au duc de Berry:

A tres noble prince et redoubté seigneur Jehan, fils de roy de France, duc de Berry et d'Auvergne et comte de pluseurs paÿs, son humble serviteur frere Jacques le Grant, religieux augustin, honeur, reverence avec toute obeÿssance ${ }^{20}$.

12 Né en 1340, le duc Jean de Berry a 70 ans au moment où il reçoit ce traité. Depuis 1392, son neveu, le roi Charles VI, est ébranlé par des crises de folie. Son frère, Philippe, duc de Bourgogne, est mort en 1404 ${ }^{21}$. Le duc de Berry reste le seul survivant des fils de Jean II, ce qui explique peut-être la dédicace de l'augustin. Le traité eut du succès: on compte plus de soixante-dix témoins manuscrits, dont le Condé 297. Le destinataire de cette belle et tardive copie n'est pas identifié.

13 L'ouvrage est un traité moral, par moment apparenté au genre des miroirs du prince ${ }^{22}$. Il comprend deux parties : la première est consacrée « au remede qui est contre les sept pechiéz mortelx » et la seconde « parle de trois estas, c'est assavoir des gens d'Eglise, de l'estat des princes et de l'estat du commun peuple». Le religieux de l'ordre de saint Augustin est visiblement très préoccupé par le péché capital d'avarice, mais nulle part dans ce traité, il n'est question du paiement d'un impôt au roi. Si cette miniature ne représente pas un prince recevant « l'impôt or de ses sujets », que représente-t-elle?

Les premiers mots du folio inscrits au-dessous de la miniature permettent de la situer dans ce miroir: «Le prince doibt estre comme le chief lequel doibt tous les... ». La miniature correspond au chapitre VIII du second livre intitulé : «Comment les princes ne doivent estre convoiteux ne avaricieux $»^{23}$. Sans imaginer qu'il y a un lien illustratif 
absolu entre texte et image, l'analyse du chapitre VIII apporte des éléments explicatifs. Comme dans l'ensemble du traité, Jacques Legrand multiplie les références à des autorités bibliques, antiques - et plus particulièrement romaines -, sans omettre les Pères et les grands ouvrages, tel le Policraticus de Jean de Salisbury. Le prince doit être le chef qui protège et non celui qui s'empare des biens des sujets. Il cultivera la vertu de libéralité qui le fera aimer de son peuple. L'auteur alterne modèles et contre-modèles : Titus ou Alexandre n'ont jamais refusé de donner, alors que le roi Antigone s'abstint et déclara que les grands dons ruinaient le royaume et que les petits déshonoraient le prince. Le gouvernant doit néanmoins se garder de folle largesse, ajoute-t-il, même si ce défaut est moins fréquent que la hideuse convoitise. L'augustin reprend alors une variante de l'apologue des membres et de l'estomac, très intéressant pour tout historien de l'impôt. L'apologue raconte que les membres, las de nourrir l'estomac, cessèrent de l'alimenter; le corps défaillit et les membres comprirent l'importance de chaque organe dans la survie du corps ${ }^{24}$, car l'estomac reçoit la « viande » (impôt), il n'en retient pour lui "fors tant seulement sa nourriture » (entretien de son Hôtel), et distribue tout le reste aux membres ${ }^{25}$.

Jacques Legrand relate ensuite les histoires édifiantes, racontées par Valère Maxime, de plusieurs vertueux Romains qui refusèrent honneurs et richesses pour prix de leur peine ${ }^{26}$. Chaque histoire lui donne l'occasion de développer des phrases d'allure proverbiale faciles à retenir, comme "c'est plus belle chose donner que recevoir » ou "mieulx valoit soi gouverner par franchise que par convoitise " ${ }^{27}$. Mais le temps présent, ajoute-t-il, est plein de princes « convoiteux » auxquels il faut donner le nom de tyran. Malheur à ceux qui prennent la «chevance» des pauvres gens, Dieu leur fera rendre justice! Les hommes sont-ils pires que les bêtes qui ne se dévorent pas entre elles? Le religieux termine ce chapitre par une dernière référence :

[On] doit prandre exemple a Tyberius du quel nous lisons es croniques comment ses officiers lui conseilloient qu'il ordenast subsides et truhages sur son peuple, et il respondi que bon pasteur ne doit pas devorer ses brebis, mais nourrir et garder ${ }^{28}$.

C'est dans les Histoires contre les païens (418) d'Orose que Jacques Legrand est allé puiser cet exemple qui se trouvait déjà dans Tacite et Suétone ${ }^{29}$. La réputation de l'empereur Tibère (14-37) est alors bien établie, et on lui prête cette invitation à la sagesse fiscale : « le bon pasteur tond ses brebis mais ne les écorche pas ».

Quelle est l'histoire qui, parmi toutes celles que l'auteur a énumérées dans ce chapitre, sert de point de départ au thème de la miniature ? Les Romains, Alexandre ou Tibère ? Leurs attitudes sont identiques : ils refusent tous de recevoir de l'argent, qu'il s'agisse du prix de la rançon (Romains), du don de Darius (Alexandre) ou de l'impôt (Tibère). Il semble bien pourtant que l'image se rapproche davantage de l'histoire exemplaire de Tibère ${ }^{30}$.

La miniature ne montre donc pas un prince recevant "l'impôt or de ses sujets », mais un prince refusant l'impôt, qu'il ne saurait lever, ou les cadeaux, qu'il ne peut accepter. Le geste du roi est un signe de refus et non d'acceptation ${ }^{31}$. Ses paumes repoussent l'or présenté sur les assiettes ou suggéré par la rondeur de la bourse. Le sage roi se tient devant un château, symbole de son pouvoir temporel défensif. Les trois sujets riches et bien mis montrent implicitement la prospérité du royaume. La miniature illustre le désintéressement du prince qui ne gouverne pas pour son propre bien, mais pour celui de ses sujets. L'image participe, à sa manière, aux débats sur la légitimité du prélèvement fiscal et la nature du lien financier unissant le roi aux sujets. 
Quelle récompense pour le prince?

damnation du tyran. Le développement arrive dans les ouvrages au terme de l'énumération des vertus et des vices. Quelle sera la récompense du roi ? Les sources antique et biblique oscillent entre l'affirmation de la gratuité du service et la nécessité de rendre à chacun son dû.

La gratuité du service

C'est une idée ancienne chez les Grecs que l'activité politique est gratuite. Dans le premier livre de la République, consacré à la justice, Platon relate une discussion entre Socrate et Thrasymaque sur les vrais mobiles du gouvernant ${ }^{32}$. Il développe la comparaison, classique dans la littérature politique, entre berger, médecin et prince. Le berger ne se conduit pas en pasteur mais en mercenaire quand il engraisse ses moutons pour en tirer profit.

De même le gouvernant n'est pas un gouvernant quand il s'enrichit aux dépens des sujets. Le médecin, le berger, le gouvernant procurent ce qui est avantageux au sujet commandé. Les gens de bien ne veulent gouverner ni pour les richesses ni pour les honneurs: ils ne veulent pas être traités de mercenaires en exigeant ouvertement le salaire de leur fonction, ni de voleurs, en tirant d'eux-mêmes de leur charge des profits secrets ${ }^{33}$.

L'opposition est clairement établie entre le mercenaire et le gouvernant. Le savoir pratique (art) du mercenaire lui assure un salaire. Il travaille pour lui-même. En revanche, l'art du médecin ou du capitaine procure le bien au sujet commandé. Ainsi le praticien apporte la santé, et le pilote, la sécurité de la navigation. L'objet du gouvernant est le bien des sujets, comme la santé est le but du médecin, et le salaire celui du mercenaire. Dans la cité idéale de Platon, philosophes et guerriers assument leurs tâches avec une totale abnégation ${ }^{34}$.

La question de la récompense du prince est néanmoins posée. C'est parce que le service est gratuit qu'un pretium doloris était attaché à l'exercice du gouvernement. Il s'agissait d'un dédommagement pour le poids (onus) de la charge qui relevait d'un devoir de justice ${ }^{35}$. La gratuité du service, prônée plus haut, et le devoir d'accorder un salaire au gouvernant, affirmé ici, peuvent sembler contradictoires. Il faut comprendre que Platon distingue l'objet du métier, qui sera le bien des moutons ou celui des sujets, et le service du travailleur, c'est-à-dire la peine qu'il a prise pour exercer cet art. Cette distinction est fondamentale ; on la retrouvera lors des discussions sur la légitimité du salaire des prédicateurs, des maîtres ou même du roi.

Le mot «salaire » est sans doute un peu fort pour qualifier l'indemnité des magistrats (misthos) que Périclès introduit à Athènes entre 454 et $450^{36}$. Cette rémunération des activités publiques, financée par le tribut des Alliés, était une obole quotidienne équivalente au minimum vital prévu pour un soldat ${ }^{37}$. Le choix de cette référence soulignait au passage les similitudes entre le service civil et le service militaire. Dans l'Éthique à Nicomaque, Aristote confirme le désintéressement du gouvernant :

Le roi est, par définition, un être complètement indépendant. [...] Il ne saurait s'intéresser à ce qui lui est utile personnellement, mais seulement à ce qui peut servir aux autres sujets ${ }^{38}$.

La tradition chrétienne s'inspire fortement de l'enseignement des Anciens. Le prince tire sa récompense de la paix qui règne dans le royaume, c'est-à-dire du résultat atteint par sa fonction. Le prince n'est pas un mercenaire, il ne saurait être question pour lui de gagner des biens matériels pour l'exercice d'un pouvoir qui tient à la fois du 
temporel et du spirituel. Le péché de simonie est sous-jacent. Cependant la récompense est due au roi comme un fait de justice ${ }^{39}$.

Thomas d'Aquin consacre quatre chapitres du De regno (1265-1267) à la récompense du roi (I, 7-10). Ce dernier étant dans son royaume comme Dieu dans le monde, nulle récompense terrestre ne saurait assouvir les aspirations royales ${ }^{40}$. La gloire est éphémère et c'est un salaire (merces) bien fragile, entièrement dépendant de l'humeur souvent changeante des hommes ${ }^{41}$. Les récompenses (praemia) temporelles ne servent qu'à exaspérer la cupidité du prince qui souhaitera posséder toujours plus de biens,

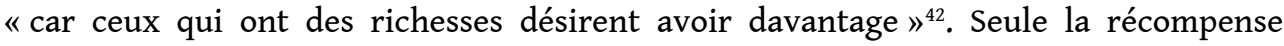
spirituelle, de même essence que l'origine et la fin du pouvoir politique, est en mesure de combler l'âme du roi soucieux du bon gouvernement du royaume. Le roi sert Dieu par son gouvernement, c'est de Lui qu'il doit recevoir la récompense pour son service : «Dieu seul peut apaiser le désir de l'homme et le rendre heureux, et être une récompense (praemium) convenable pour le roi ${ }^{43}$. Seule la Béatitude peut assouvir les aspirations de l'homme. La gratuité de l'office royal est affirmée. Il n'y a pas de conversion matérielle possible des bienfaits rendus. Thomas d'Aquin suit l'enseignement de l'Écriture et des Pères: l'homme ne trouvera jamais de récompense satisfaisante ici-bas ${ }^{44}$.

Le gouvernant ne sert pas pour de l'argent, et certainement pas avec l'argent de ceux qu'il sert. Le prince n'a pas été institué pour son propre gain (propria lucra) ${ }^{45}$; il exerce une fonction ministérielle et assure lui-même les moyens du service. Le vocabulaire de la rémunération (merces, praemium) est ici employé dans un sens spirituel, dans un jeu de comparaison, habituel chez les théologiens, entre les domaines spirituel et temporel. Dans le contexte du développement de la prédication et des Universités, certains vont néanmoins essayer d'exposer une réflexion conciliant gratuité et rémunération pour les «ministres » des deux charges. La résolution de cette contradiction, bien dans le goût des scolastiques, permet d'aborder la question du « salaire du prince ».

La rémunération des maîtres et le "salaire du prince »

L'analyse de la rémunération des clercs, des prédicateurs et des maitres va servir de contrepoint à l'étude du "salaire du roi », l'essor de ces activités posant en effet le problème de leur rémunération. La Bible étant le miroir du monde, tous allaient $\mathrm{y}$ puiser un enseignement. Or les nombreuses occurrences bibliques du thème du travail et de la rétribution sont loin d'être cohérentes. L'Écriture enseigne à la fois qu'il faut donner gratis et ne rien attendre en retour (Mt 10,9), et que nul ne combat à ses propres frais $(1$ Cor 9,7$)$.

28 L'héritage judéo-chrétien rapporte l'enseignement d'un Dieu actif, créateur (operatio) qui se repose le septième jour (Gn 2,1), et d'un homme (Adam) qui cultive (operare) le jardin d'Éden (Gn 2,15) jusqu'à la chute qui le condamne à travailler la terre pour gagner son pain à la sueur de son front (Gn 3,16-19). Quel fut l'exemple du Christ et des apôtres ? Le Christ est le fabri filius. Lui-même ne travaille pas, mais il n'est pas inactif : il prêche aussi bien l'abandon à la Providence et l'exemple des lys des champs qui ne travaillent pas (non laborant) que les injonctions au travail et les dénonciations de l'oisivetét ${ }^{46}$. Le Christ et les disciples ont de riches amis (Joseph d'Arimatie, Nicomède, Simon le Pharisien, Jeanne, la femme de Chouza, l'intendant d'Hérode) qui les assistaient de leurs biens (quae ministrabant eis de facultatibus suis) ${ }^{47}$. Le Christ dispose d'une bourse (loculus), tenue par Judas, mais l'Écriture précise que le Seigneur ne s'en servait pas pour payer l'impôt (Mt 17, 24-27) ${ }^{48}$. Quant aux apôtres, les épîtres 
pauliniennes témoignent de la complexité de leur vie de prédicateur. La rémunération est un dû, chacun doit vivre de son ministère $(1 \text { Cor } 9,13)^{49}$, mais l'Apôtre la refuse pour lui-même, pour plusieurs raisons. La première est la nécessité d'être libre : Ne suis-je pas libre? (1 Cor 9, 1), libre à l'égard de tous (1 Cor 9, 19), libre de se consacrer complètement à sa tâche apostolique. "L'apôtre ne voulut pas user du pouvoir que le Seigneur lui avait donné et recevoir son entretien (stipendia victus) de ceux à qui il prêchait l'Évangile du Christ, de peur de faire obstacle à celui-ci $\aleph^{50}$. La deuxième raison relève davantage d'une réflexion philosophique: Paul remplit une tâche en évangélisant, il offre sa prédication sine sumptu. Sa récompense est dans le bienfait apporté aux populations, comme la santé était la récompense du médecin. Le paradoxe de ce passage explique en partie les atermoiements futurs sur la rétribution des prédicateurs. Récapitulons l'enseignement de l'Apôtre: la rémunération pour une peine est un devoir de justice, mais Paul n'use pas de ce droit, il répand la parole avec libéralité, c'est-à-dire qu'il n'exerce pas, de fait, son droit.

Les réflexions parallèles entre la rémunération royale et l'entretien des prédicateurs ou des maitres ne sont pas fortuites puisque ce sont les théologiens eux-mêmes qui établissent des rapprochements entre la rétribution des ministres du culte et celle des ministres du bien commun. Thomas d'Aquin et Gilles de Rome opèrent une double comparaison entre dîmes et cens, d'une part, et ministres du culte et serviteurs de l'utilité commune, d'autre part. Pour Gilles de Rome, la dîme est une obligation d'entretien du clergé, car ce dernier assure les services spirituels pour le salut du peuple.

Les dîmes sont comme le cens (quasi census) de nos possessions, et les offrandes sont comme les tributs (quasi tributa) que nous donnons à Dieu et à l'Église, tant sur nous que sur nos biens, en reconnaissance de notre propre servitude, car on voit que certaines lois disent que ces mêmes dîmes sont comme des tributs ${ }^{51}$.

30 Le peuple doit pourvoir à l'entretien des clercs et des princes, précise Thomas, dans la Somme théologique ${ }^{52}$. Il reprend la même comparaison entre les ministres de Dieu et les serviteurs du bien commun dans la question : trouve-t-on dans l'Ancien Testament des principes convenables concernant les gouvernants $?^{53}$ Le dominicain rappelle que la loi a assigné aux prêtres et aux lévites les moyens d'assurer leur subsistance, mais que rien n'a été prévu pour l'entretien des princes. En effet, les prêtres venaient de la tribu de Lévi, c'est-à-dire de la douzième tribu, celle qui n'avait pas reçu de propriété, il fallait donc leur trouver des revenus : ce seront les dîmes. En revanche, les rois sont choisis au sein de la population; ils devront disposer de biens propres (certas) dont ils pourront vivre. Pour les deux théologiens, l'obligation de contribuer à l'entretien des ministres découle du droit naturel permanent. C'est le bien commun, auquel œuvrent princes et prêtres, qui justifie leur rémunération.

31 La croissance du nombre des prédicateurs et le développement de l'Université ont suscité deux problèmes : l'un, théologique et l'autre, pratique. D'une part, la question se posait de la légitimité du "salaire » temporel accordé à des maîtres qui rendaient un service spirituel. D'autre part, un déséquilibre menaçait de se former entre le nombre des clercs et la quantité de biens attachés à leur entretien. Les réponses apportées par les canonistes à propos de la rétribution des maîtres présentent quelques points communs avec cette recherche menée sur le « salaire du roi ».

Les travaux de Gaines Post mettent en évidence la méfiance des Anciens, des Pères de l'Église et des juristes à l'égard de la rémunération des maîtres ${ }^{54}$. À tous, il apparaît que 
vertu et richesse sont antinomiques. Le discours était à la fois convenu et imprécis, car le salaire du maître relevait davantage de la subsistance que de la richesse. Certains canonistes du xiiie siècle, comme Johannes Teutonicus dans son Apparatus (ca 1215-1217), exposèrent que chaque école cathédrale devait appointer un maitre et le pourvoir d'un bénéfice pour enseigner gratis ${ }^{55}$. Ainsi, les maîtres des Universités et des écoles cathédrales ayant des bénéfices ecclésiastiques ne devaient rien demander aux pauvres écoliers. Ils pouvaient en revanche accepter des honoraires de ceux qui étaient riches. Et si les maitres n'avaient pas de ressources, ils pouvaient recevoir des honoraires de tous, sauf des pauvres. La typologie des situations montre bien que tout dépendait de l'adéquation bénéfices-office; de plus, il était bien précisé que le maître ne devait pas demander, mais qu'il pouvait éventuellement accepter des honoraires. Le maître ne demandera une contribution à ses élèves que si ses ressources sont insuffisantes. Cette réglementation est parente de la justification de l'impôt royal : le prince ne peut imposer ses sujets que si ses ressources sont insuffisantes.

Le problème pratique étant résolu, il fallait en venir au problème spirituel, car l'enseignement est d'une double nature. Dans son Commentaire des Sentences, Thomas d'Aquin explique qu'un acte est spirituel, soit parce que son principe l'est, soit parce que le produit est spirituel ${ }^{56}$. Dans le premier sens, il y a risque de simonie s'il y a monétarisation des services, mais on peut accepter une sustentationem vitae. Dans le second cas, la vente est possible. Ainsi, le maître peut monnayer sa peine (labores suos), mais non le produit spirituel de son travail, c'est-à-dire le savoir ou la vérité, car la sagesse et la grâce sont des dons de Dieu, et ne peuvent être vendues (scientia donum Dei est, unde non vendi potest). La distinction établie par les théologiens entre la science du maître, qui vient de Dieu, et le travail du maitre, fruit de ses capacités pédagogiques, permit d'envisager la rémunération temporelle. Cette dernière s'apparentait davantage à un dédommagement matériel, destiné à l'entretien du pédagogue, qu'à un véritable salaire. Deux arguments avaient dénoué la situation : le premier rappelait l'origine divine de la science qui interdisait qu'elle puisse être vendue, à moins de commettre un vol puisque le maître n'en était pas propriétaire; le second mettait l'accent sur le rapport dissymétrique entre le savoir et l'argent qui $n$ 'ont pas de commune mesure, car le savoir est inestimable ${ }^{57}$. Ce n'est donc pas le savoir qui était vendu, mais le savoir-faire. Le même problème se posait, à des titres différents, pour les prédicateurs et même pour les marchands. Dans chacun des cas, il s'agissait de distinguer premièrement, science et labeur, et deuxièmement, d'éviter de réclamer la rémunération. Si les princes exerçaient une fonction parfois assimilable aux ministres du culte, n'était-ce pas un devoir de justice d'envisager une rémunération à leur endroit?

L'impôt est-il le « salaire du prince »?

Le pouvoir du roi est longtemps resté principalement négatif et conservatoire. Le roi était le minister : il était l'instrument d'un pouvoir qui ne lui appartenait pas, car tout pouvoir vient de Dieu $(\mathrm{Rm} 13,4)$. Le roi garde la paix et maintient l'ordre (pax et ordo). Le roi protège le peuple, le nourrit, tel le Bon Pasteur qui paît ses brebis (Jn 21, 17). Il inspire la crainte aux méchants, et si celle-ci ne suffit pas, il punit ceux qui font le mal. Dès lors, les dépenses d'un tel roi sont forcément modérées, car l'étendue de son pouvoir est limitée, aussi peut-il vivre de ses revenus ordinaires. L'évolution de la fonction royale transforma fondamentalement les besoins financiers du prince et entraîna une modification de l'argumentation de l'impôt. 
L'évolution de la fonction royale d'acquitter l'impôt pour soutenir la majesté royale et la richesse du royaume. Ainsi le franciscain Nicolas de Lyre (†1349), dont on connaît la foi monarchique, fait de la pompe royale un cas de nécessité au même titre que la défense du royaume (solum ad necessitatem sui status et regni defensionem ${ }^{61}$. L'exégèse des très significatifs versets de Dt 17 (Qu'il n'aille pas multiplier ses chevaux [...], le nombre de ses femmes [...], son argent et son or) évolue : les commentateurs du xive siècle font primer la sécurité du royaume (necessitas) sur le risque moral (superbia) couru par un prince trop puissant ${ }^{62}$. La magnificence du roi est un signe, visible aux yeux de tous, de l'utilisation de l'impôt. Cette visibilité le rend plus supportable et contraste absolument avec l'invisibilité suspecte des dépenses administratives.

L'évolution de la fonction royale se manifeste également, dès avant le xiiie siècle, par la valorisation du travail du prince, perceptible dans la littérature des miroirs. Tous le répètent à l'envi après Guillaume de Malmesbury, et surtout depuis que Jean de Salisbury en a fait une maxime politique dans son Policraticus (1159) : « un roi illettré est comme un âne couronné $\star^{63}$. Le roi doit être vertueux et sage. Rien ne doit le distraire de son ministère. L'épître de Paul (1 Cor. 1-2) permettait de jouer sur les deux acceptions divine et humaine de la sagesse, qui ne s'opposent pas nécessairement. Christine de Pizan (ca 1364-ca 1429) et Jacques Legrand ont mis en avant la sagesse humaine du prince dans leurs écrits, sagesse dont ils ont vu une incarnation dans la personne du roi Charles $\mathrm{V}^{64}$. La majeure partie du Livre des faits et bonnes mœurs du roi Charles $\mathrm{V}$ de Christine est consacrée à la culture et la sagesse du souverain. La sagesse garantit la prospérité et la durée du royaume (III, 14). L'insistance à vanter le savoir, ainsi que les nombreuses représentations du monarque dans sa bibliothèque, impriment à l'image idéale du prince un changement très notable. Elle découple le roi 
de ses fondements essentiels du sang et du sacre. De plus, il n'est pas évident que l'idéal du sage Charles V soit toujours en adéquation avec l'idéal des populations.

L'office royal est une lourde charge dont les sujets doivent être conscients. Son travail (labor, studium) et sa peine sont le prix de la paix des sujets, expliquait Jean Chrysostome ${ }^{65}$. Pour Thomas d'Aquin, le fardeau de l'office royal (onerosum regis officium) coûte au prince beaucoup de peine et de soucis (si tot labores et sollicitudines) ${ }^{66}$. Le dominicain Guillaume Peyraut revient lui aussi sur le «fardeau accablant» (onus importabile) pesant sur les épaules du roi ${ }^{67}$. Le poids du service accapare le temps, les richesses, la vie même du prince qui néglige ses propres affaires pour s'occuper des affaires publiques. Entièrement tourné vers la fin de sa fonction, le gouvernant s'oublie lui-même dans l'accomplissement de sa charge. Le souci (sollicitudo) que prend le prince à maintenir la paix justifie la récompense matérielle qu'il reçoit ${ }^{68}$. Vu du côté des gouvernés, l'office royal est perçu comme un bienfait dont ils doivent se montrer reconnaissants. La vue d'une cité pacifiée n'est-elle pas la preuve du paiement de l'impôt par les citoyens reconnaissants? Alors que les sujets dorment et reposent, le prince veille sur leur sécurité ${ }^{69}$. L'effet (la paix) permet de remonter à la cause (rétribution du labeur des gouvernants). Les princes servent l'utilité publique à laquelle ils ne sont pas tenus gratis. Cette formulation elliptique termine la réponse $\mathrm{du}$ maitre en théologie Berthaud de Saint-Denis à une question qui lui fut posée en 1282 sur l'acquittement de l'impôt par les clercs : les princes ne sont pas tenus gratuitement (gratis) à la défense et à la protection des clercs et des églises ${ }^{70}$. La nature du lien financier qui s'établit alors entre le prince et ses sujets a été diversement expliquée par les « gens de savoir »"

La nature du lien financier existant entre le prince et les sujets

L'affirmation du pouvoir royal, le poids plus lourd de l'impôt et sa monétarisation croissante ont incité les contemporains, théologiens et canonistes pour la plupart, à poser la question de la nature de l'impôt. Est-il un signe de soumission, une avance avant remboursement, un dédommagement pour le prince ou le «salaire du roi »? Le dernier thème peut surprendre mais les théologiens du xiiie siècle l'évoquent et l'envisagent dans les commentaires du chapitre 13 de l'épître aux Romains sur l'origine du pouvoir royal, qui s'achève par Rendez à chacun ce qui lui est dû : à qui le tribut, le tribut; à qui le vectigal, le vectigal $(\mathrm{Rm} 13,7)$.

41 L'étude comparée d'une soixantaine de commentaires de $\mathrm{Rm} 13$, datant du ix ${ }^{\mathrm{e}}$ au xiv siècle, permet de constater la permanence d'un fond de thèmes communs (soumission au pouvoir) et l'apparition de certains thèmes nouveaux, telle la rétribution du prince $^{72}$. C'est au xiiie siècle qu'elle fait son entrée thématique dans l'exégèse universitaire de $\mathrm{Rm} 13^{73}$. On la trouve principalement représentée dans l'exégèse mendiante, toujours plus sensible aux sujets financiers.

Les commentateurs s'attachent à expliquer les premiers mots du verset 6 : En effet, vous fournissez les tributs (Ideo enim et tributa praestatis) car les ministres sont les serviteurs de Dieu en cela même qu'ils s'appliquent de par Dieu à cet office ${ }^{74}$; en particulier, le verbe praestare. Les tributs sont "fournis " et non pas "acquittés ", explique le franciscain Jean de la Rochelle $(\dagger 1245)$, ce qui tend à montrer que ce paiement est volontaire et non coercitif. L'acquittement est présenté comme un équivalent financier de la défense de la patrie et du maintien de la justice par les princes $^{75}$. Le tribut est fourni en retour du bon gouvernement. C'est librement que les gouvernés s'acquittent des tributs puisque le prince veille au bien du royaume. 

et Pierre de Tarentaise (†1276) s'appliquent encore plus précisément à décortiquer le sens et la nature du lien financier existant entre gouvernant et gouverné. Le commentaire de Thomas, qui date des dernières années de sa vie $(1272-1273)^{76}$, revient à deux reprises sur l'impôt comme solde du prince.

Par conséquent, puisque nos princes dans leur gouvernement servent (ministrent) Dieu, ils doivent percevoir les tributs du peuple, comme solde de leur ministère (quasi stipendia sui ministerii). [...] Les tributs (tributa) sont dus aux rois comme la solde du travail (quasi stipendium laboris) ${ }^{77}$

La formulation quasi stipendium laboris a de quoi surprendre. Seul l'emploi de la conjonction quasi atténue un peu la portée de la comparaison entre l'impôt et la solde du travail royal. Thomas d'Aquin qualifie l'impôt de "solde », le point est déjà intéressant, mais il y a mieux : le dominicain ajoute « solde du travail ». Il ne semble pas distinguer, comme l'ont fait d'autres théologiens à propos de la rétribution des maîtres, le « travail » qui est gratuit et le « savoir-faire » qui peut recevoir une rétribution. Tarentaise, va plus loin. Il se présente sous le modèle scolastique en vigueur : question, arguments " pour ", " contre » et « solution".

Servants en cela; la glose [dit] : «c'est-à-dire pour le tribut »; Contre : donc la récompense $\mathrm{du}$ prince est temporelle (praemium principis est temporale). Je réponds: le tribut n'est pas la récompense du travail (tributum non est praemium laboris), mais la solde du travailleur (sed stipendium laborantis) ${ }^{78}$.

Fidèle à l'enseignement des maîtres qui ont su résoudre le problème du salaire des maîtres par une différenciation entre savoir et savoir-faire, Pierre de Tarentaise distingue le travail (labor) et le travailleur (laborans). La solde (stipendium) est le prix de la peine du travailleur et non pas la récompense du travail ${ }^{79}$. Pierre de Tarentaise se montre beaucoup plus attentif que Thomas d'Aquin à la séparation de ces deux registres. Le ministère royal est gratuit, mais la peine prise par le prince mérite une solde. Le débat contemporain sur la rémunération des maîtres et des prédicateurs n'est certainement pas étranger à la position prise par Pierre de Tarentaise.

À la lecture des commentaires universitaires de Rm 13, il ressort que l'acquittement des tributs est directement lié à l'exercice des fonctions du prince. Les exégètes ont diversement interprété la nature de ce lien de causalité. Pour certains, l'acquittement des tributs s'apparente à une restitution. Les tributs ne sont pas "payés ", mais « fournis » en retour des frais engagés par le prince pour maintenir la paix et la justice dans le royaume, car le roi ne doit pas s'appauvrir ${ }^{80}$. L'impôt contribue à maintenir le niveau de son trésor qui doit rester stable ou augmenter, mais certainement pas baisser ${ }^{81}$. Pour d'autres, l'impôt est envisagé comme la solde du travail ou du travailleur (stipendium laboris/laborantis). Le vocabulaire (stipendium) assimile le prince au soldat; comme lui, le prince rend un service, qui est d'ailleurs, pour une part, un service militaire.

L'impôt est-il le «salaire du roi »? Pour la forme, on notera chez la plupart des théologiens l'identité de vocabulaire pour désigner le salaire du maître, du prédicateur, du marchand ou du prince. Quel que soit le domaine, cette rétribution est désignée sous le nom de stipendium laboris. L'emploi de métaphores militaires et laborieuses par les exégètes ne simplifie pas les interprétations. La confusion du vocabulaire ne doit cependant pas nous abuser. Sur le fond, une distinction est faite entre la rétribution de 
la peine et la gratuité du service. Pierre de Tarentaise l'établit de manière très convaincante (non est praemium laboris sed stipendium laborantis).

Ainsi, le roi, vu par les théologiens, reçoit deux récompenses. Il perçoit des sujets une aide financière légitime, que certains qualifient de rétribution, de compensation ou de remboursement, mais le prince n'est pas comme le mercenaire de la parabole qui s'enfuit lorsque survient le danger (Jn 10,22-38), le roi est comme le Bon Pasteur qui recherche le bien de ses brebis et se dévoue pour elles. Le service du roi et l'impôt relèvent de deux registres différents, l'un est spirituel ou apparenté au ministère spirituel, l'autre est temporel. Le sujet ne pourra jamais rendre au prince l'équivalent de la pax régnant dans le royaume ; il se trouve sur ce point dans la même situation que l'enfant vis-à-vis de ses parents qui ne pourra jamais leur rendre la vie qu'ils lui ont donnée. L'échange est inégal et dissymétrique. La conversion des deux est impossible.

La reconnaissance - dans les deux sens du mot - du travail du roi ne remet pas en cause la gratuité du service, même si elle permet d'envisager une rémunération. La royauté est à la fois une dignité, un pouvoir et un office. La complexité de la nature du pouvoir royal permet d'envisager plusieurs types de récompenses. Le roi exerce un service qui est à la fois "gratuit et rémunéré ».

51 Au regard de ces éléments, on comprend que le roi de la miniature du Livre de bonnes meurs ne peut accepter les plats et la bourse qui lui sont tendus. Le lien qui unit le roi aux sujets n'est ni apparenté au "don-contre-don " $^{82}$, qui est un échange obligé, ni à un contrat délimitant les obligations réciproques, mais à une forme d'union "gracieuse " ou gratuite, de type christique. Que cet or provienne de l'impôt ou de dons, le roi ne peut les recevoir. Ces pièces sont probablement le signe de la gratitude des sujets pour leur prince. Le roi pourrait recevoir l'impôt ou les dons, de droit - la théorie fiscale justifiant l'impôt existe -, mais il montre son élévation morale en les repoussant de fait, ce qui apparente son attitude à celle de l'Apôtre (1 Co 9). La vraie récompense terrestre du prince réside dans le bon ordonnancement du royaume, visible sur cette image. L'enseignement moral du Livre de bonnes meurs est certes d'une grande richesse spirituelle, mais il est porteur de nombreuses difficultés matérielles à venir pour le prince. Il n'y a pas dans cette miniature de lien de nature financière entre le roi et ses sujets. Il en existe d'autres, implicitement représentés : vu du côté du prince, le château symbolise la protection contre les ennemis; vu du côté des gouvernés, l'attitude des trois hommes montre leur déférente attention au roi. La miniature du Livre de bonnes meurs ne représente pas le roi levant «l'impôt or sur ses sujets ». Proposons la légende suivante : « Le roi refuse l'or de ses sujets ».

Alors que le prélèvement fiscal est largement en place dès le début $\mathrm{du} \mathrm{xv}^{\mathrm{e}}$ siècle, le Livre de bonnes meurs reste fidèle à l'idéal ministériel. Jacques Legrand (1410) et l'artiste du manuscrit du fonds Condé (1490) semblent plus attachés à l'aspect moral de la fonction du prince qu'à sa réalité politique ou financière. Faut-il le qualifier d'archaïque ? Le terme paraît inapproprié sauf à désigner ainsi tous les traités - et ils sont nombreux - qui restent fidèles à cet idéal. Le prédicateur Jacques Legrand connaît bien les débats concernant l'impôt ; ses sermons relatés par le Religieux de Saint-Denis en témoignent. Mais les artistes comme les auteurs de miroirs hésitent à mettre en scène des rois levant l'impôt. La représentation de la levée fiscale va contre la nature même de la conception royale, telle que roi et sujets souhaitent la voir exprimée. Le roi n'est pas un loup rapace qui effraye le troupeau dont il a la garde. De leur côté, les sujets ne sont pas des moutons que l'on peut tondre jusqu'au sang. Nul ne souhaite 
l'existence de telles images. Alors que la réalité financière du pouvoir s'accentue, seule la représentation du don gratuit, c'est-à-dire gracieux et libre, est en mesure de montrer la part affective qui unit les différentes parties du corps politique.

\section{NOTES}

1. Le manuscrit offert au duc de Berry est le ms Paris, BnF fr. 1023. Il porte la date du 4 mars 1410. La scène de dédicace est illustrée par une miniature au fo $2 \mathrm{r}^{\circ}$. Sur le BnF fr. 1023, voir A. Châtelet, « Un traité de bonnes mœurs écrit et illustré dans une période tourmentée : le manuscrit français 1023, daté de 1410 de la Bibliothèque nationale de France ", Art de l'enluminure, 1, 2002, p. 40-62.

2. Jacques Legrand, Le Livre de bonnes meurs, ms. Chantilly, Condé 297. La miniature qui nous intéresse se trouve au fo $79 \mathrm{v}^{\circ}$.

3. Le Livre de bonnes meurs connut un grand succès : 73 manuscrits connus, copiés pendant tout le Moyen Âge ; 13 éditions anciennes ; 3 traductions anglaises indépendantes et une retraduction en latin en 1507. Jacques Legrand, Archiloge Sophie. Livre de bonnes meurs, éd. E. Beltran, Paris, 1986, p. 287-295.

4. Les débats sur la fiscalité royale portent essentiellement sur l'impôt direct, beaucoup plus que sur les taxes indirectes (aides, gabelles...).

5. E. A. R. Brown, «Cessante causa and the Taxes of the Last Capetians : the Political Application of a Philosophical Maxim », Studia Gratiana, 15, 1972, p. 565-588.

6. Jehan Masselin, Journal des États généraux de France tenus à Tours en 1484 sous le règne de Charles VIII, éd. A. Bernier, Paris, 1835, p. 449.

7. L. Scordia, Le roi doit vivre du sien. La théorie de l'impôt en France (XIII $-X V^{e}$ siècles), à paraître aux Étude Augustiniennes.

8. Chiffres donnés par Ph. Contamine, « Réformer l'État, rationaliser l'administration : à propos du contrôle des finances publiques vers 1456-1461 », Finances, pouvoirs et mémoire. Hommages à Jean Favier, textes réunis par J. Kerhervé et A. Rigaudière, Paris, 1999, p. 388-396, à la p. 390.

9. Sur cette miniature, les officiers de la Chambre des Comptes sont assis autour d'une table recouverte d'une toile verte - qu'on appelle alors un « bureau »-, sur laquelle ils comptent les pièces et enregistrent les chiffres dans des livres ou sur des rouleaux.

Henri Baude, De la vie, condition et complexion du roi Charles VII, Paris, BnF, lat. 6222 C, fo $42 v^{\circ}$; voir C. Beaune, Le Miroir du pouvoir, Paris, 1989, p. 107.

10. Pierre Choisnet, Le Livre des trois aages, Paris, BnF, fr. Smith Lesouëf $70, f^{\circ} 9 v^{\circ}$.

11. Cette miniature accompagne la traduction de la Cité de Dieu d'Augustin par Raoul de Presles. Voir A. de Laborde, Les manuscrits à peinture de la Cité de Dieu de saint Augustin, Paris, 1909, t. 1, p. 231-235 ; J. Baschet, Les justices de l'au-delà. Les représentations de l'Enfer en France et en Italie (XII $-X V^{e}$ siècles), Rome, 1993, fig. 139.

12. L. Delisle et G. Macon, Chantilly. Le cabinet des livres manuscrits, Paris, 1900, t. 1, p. 241-242; J. Meurgey, Les principaux manuscrits à peintures du musée Condé de Chantilly, Paris, 1930, p. 101-102. 
13. La miniature illustre la rubrique « impôt » du Dictionnaire encyclopédique du Moyen Âge (DEMA), dir. A. Vauchez, Paris, 1997, t. 1, p. 769.

14. Le manuscrit est relié en maroquin citron à tranche dorée avec filets et petits fers. La reliure date du xvii ${ }^{\mathrm{e}}$ siècle. Le dos et les plats sont semés d'hermines. Cette pièce provient de la bibliothèque des ducs de Bretagne, selon le catalogue Boorlut de Noortdonck d'avril 1858.

15. Le texte n'est pas paginé. Si l'on commence la numérotation à l'exposition du plan qui se trouve en tête de volume, le manuscrit compte 156 feuillets, et non 154, comme l'indique la notice très succincte de Chantilly.

16. Depuis quelques années, l'Institut s'attache à mettre en valeur les trésors du musée Condé. Les chercheurs de Chantilly, du département des manuscrits de la BnF et l'IRHT travaillent à faire connaître la richesse du fonds. Voir le catalogue de l'exposition tenue au printemps 2003 (commissaire : Patricia Stirnemann, avec la collaboration de Claudia Rabel) : L'enluminure en France au temps de Jean Fouquet, Paris-Chantilly, 2003. La notice du Condé 297 a été réalisée par Patricia Stirnemann, p. 48-56 et 87.

17. Les miniatures se trouvent aux fos $3 \mathrm{v}^{\circ}, 7 \mathrm{r}^{\circ}, 10 \mathrm{r}^{\circ}, 14 \mathrm{r}^{\circ}, 17 \mathrm{r}^{\circ}, 20 \mathrm{v}^{\circ}, 24 \mathrm{v}^{\circ}, 17 \mathrm{r}^{\circ}, 30 \mathrm{v}^{\circ}$, $32 \mathrm{r}^{\circ}, 35 \mathrm{r}^{\circ}, 37 \mathrm{v}^{\circ}, 40 \mathrm{v}^{\circ}, 43 \mathrm{r}^{\circ}, 47 \mathrm{r}^{\circ}, 51^{\circ} \mathrm{r}$ (avarice et libéralité), $53^{\circ} \mathrm{v}$ (scène d'avarice), $56 \mathrm{v}^{\circ}$, $61 \mathrm{v}^{\circ}, 64 \mathrm{r}^{\circ}, 67 \mathrm{r}^{\circ}, 69 \mathrm{r}^{\circ}, 71 \mathrm{v}^{\circ}, 74 \mathrm{r}^{\circ}, 77^{\circ} \mathrm{r}$ (le roi donne aux pauvres), $79 \mathrm{v}^{\circ}, 83 \mathrm{r}^{\circ}, 85 \mathrm{v}^{\circ}, 88 \mathrm{v}^{\circ}$, $91 \mathrm{v}^{\mathrm{o}}, 93 \mathrm{v}^{\mathrm{o}}, 97 \mathrm{r}^{\circ}, 99 \mathrm{r}^{\circ}, 101 \mathrm{r}^{\circ}, 103 \mathrm{v}^{\mathrm{o}}, 106 \mathrm{r}^{\circ}, 109 \mathrm{v}^{\mathrm{o}}, 111 \mathrm{v}^{\mathrm{o}}, 115 \mathrm{r}^{\mathrm{o}}, 117 \mathrm{v}^{\circ}, 120 \mathrm{r}^{\circ}, 122 \mathrm{v}^{\mathrm{o}}, 125 \mathrm{r}^{\circ}$, $127 \mathrm{r}^{\circ}, 129 \mathrm{v}^{\circ}, 132 \mathrm{v}^{\circ}, 135 \mathrm{r}^{\circ}, 138 \mathrm{v}^{\circ}, 141 \mathrm{v}^{\circ}, 144 \mathrm{r}^{\circ}, 146 \mathrm{v}^{\circ}, 149 \mathrm{v}^{\circ}, 153 \mathrm{r}^{\circ}$.

18. Jacques Legrand, Le Livre de bonnes meurs, ms. Chantilly, Condé $297, \mathrm{f}^{\circ} 79 \mathrm{v}^{\circ}$.

19. Même si le contenu du traité est largement intemporel, il ne faut pas s'y tromper, Jacques Legrand est fortement impliqué dans les affaires du temps. Les deux rédactions encadrent l'assassinat de Louis d'Orléans (1407) par Jean sans Peur. Ce contexte n'est pas à négliger. Le Religieux de Saint-Denis fait un large écho aux sermons prêchés par Jacques Legrand devant le roi, la reine et la Cour, Chronique du Religieux de SaintDenis, éd. M.-L. Bellaguet, 6 vol., Paris, 1839-1855, préface B. Guenée pour l'édition de 1994, vol. 2, XXVI (1405), 7, p. 272-273. Sur cet augustin, voir E. Beltran, « Jacques Legrand, OESA. Sa vie et son œuvre ", Augustiniana, 24, 1974, p. 132-160 et 387-414; du même, L'idéal de sagesse d'après Jacques Legrand, Paris, 1989 ; J. Krynen, Idéal du prince et pouvoir royal en France à la fin du Moyen Âge (1380-1440). Étude de la littérature politique du temps, Paris, 1981, p. 60 sq.

20. Le texte édité par Evencio Beltran est le manuscrit de Paris, BnF, fr. 1023 : Jacques Legrand, Archiloge Sophie. Livre de bonnes meurs, éd. cit., p. 300.

21. Fr. Autrand, Jean de Berry, Paris, 2000.

22. Evencio Beltran établit la généalogie complexe de ce texte. Le Livre de bonnes meurs est une partie d'un projet plus ample appelé Archiloge Sophie, qui ne fut pas mené à terme par son auteur et dont notre traité formait la fin. Ajoutons qu'il correspond à une traduction en français, avec variantes, d'une partie de l'encyclopédie de Jacques Legrand, intitulée Sophilogium, très lue jusqu'au $\mathrm{Xvi}^{\mathrm{e}}$ siècle. Jacques Legrand, Archiloge Sophie. Livre de bonnes meurs, éd. cit., p. 11-14 et 287-290.

23. Jacques Legrand, Livre de bonnes meurs, éd. cit., II, 8, p. 352-354.

24. Voir L. Harf-Lancner, «L'individu dans l'État : la fable des membres et de l'estomac dans la littérature du Moyen Âge ", L'individualisme, permanence et métamorphoses, éd. J. Attuel, Paris, 1988, p. 51-71; de la même, « Les Membres et l'Estomac : la fable et son interprétation politique au Moyen Âge », Penser le pouvoir au Moyen Âge. Études offertes à Françoise Autrand, dir. D. Boutet et J. Verger, Paris, 2000, p. 111-126. 
25. Jacques Legrand, Le Livre de bonnes meurs, éd. cit., II, 8, p. 353. Sur la métaphore de l'estomac, cf. L. Scordia, Le roi doit vivre du sien, op. cit.

26. Jacques Legrand fait référence à deux histoires édifiantes, l'une est tirée d'une série d'exempla sur la grandeur du Sénat qui repousse les rançons offertes par les Carthaginois pour récupérer leurs prisonniers $(\mathrm{V}, 1,1 \mathrm{a})$, et l'autre, sur la réputation que s'est forgée Alexandre en refusant l'argent que lui promettait Darius (VI, 4, ext. 4). On remarquera que dans les deux exemples choisis, l'élévation morale de ces modèles est d'autant plus grande qu'elle s'exerce à l'égard de leurs plus grands ennemis. Valère Maxime, Faits et dits mémorables, éd. R. Combes, Paris, 1997, t. 2, p. 68-69 et 171-172.

27. Jacques Legrand, Le Livre de bonnes meurs, éd. cit., II, 8, p. 353.

28. Jacques Legrand, Le Livre de bonnes meurs, éd. cit., II, 8, p. 354.

29. Orose, Histoire contre les païens, éd. M.-P. Arnaud-Lindet, Paris, 1991, vol. 3, VII, 4, 1, 4, p. 23 : «Ipsi autem Tiberius plurima imperii sui parte cum magna et gravi modestia reipublicae, praefuit adeo ut quibusdam praesidibus augenda provinciis tributa suadentibus scripserit "boni pastoris esse tondere pecus non deglubere" ». La référence à Orose est donnée dans l'édition d'E. Beltran, p. 412. On trouve également l'histoire dans Tacite (v. 55-v. 120), Annales, IV, 6 et dans Suétone (v. 69-v. 125), Vies des douze Césars, III, 32.

30. Jacques Legrand revient à nouveau sur le thème du refus de toute récompense quelques chapitres plus loin (II, 14) : «Comment les riches ne se doivent point glorifier en leurs richesses ». Il l'illustre par les exemples de Scipion qui « ne demanda pour son salaire nemais qu'il fut surnommé Affricain en memoire de la ditte victoire ", et celui de « Tyberius » qui « disoit que mieulx valoit tresor de souffisance que de chevance ». Les deux histoires sont tirées des Faits et dits mémorables de Valère Maxime (III, 7 ; IV, 3, 7). Jacques Legrand, Le Livre de bonnes meurs, éd. cit., p. 363.

31. Je remercie vivement $M^{\text {me }}$ Patricia Stirnemann pour les précisions qu'elle a bien voulu apporter à l'analyse de cette miniature.

32. Platon, République, éd. et trad. E. Chambry, Paris, 1932, I, 17-18. La connaissance directe de Platon est alors parcellaire (Ménon et Timée), mais il y a une grande diffusion des idées du philosophe par l'intermédiaire d'Augustin (La Cité de Dieu, V, 13 et 15-16), du Pseudo-Denys et de Boèce.

33. Platon, République, éd. cit., I, 17, 345d ; I, 18, 347a. À comparer au pastor bonus de Jn 10, 7-14 qui est prêt à donner sa vie pour ses brebis, alors que le mercenaire se désintéresse du troupeau quand survient un danger.

34. Platon, République, éd. cit., VIII, 6, 550d-550e : « N'y a-t-il pas entre la richesse et la vertu cette différence, que, placées l'une et l'autre dans les plateaux de la balance, elles prennent toujours une direction contraire?».

35. Platon, République, éd. cit., I, 18, 347a et I, 19, 347b : « C'est pour cela, me semble-til, qu'il faut assurer un salaire à ceux qui consentent à commander, soit de l'argent, soit de l'honneur, soit une punition, s'ils refusent ".

36. Alterneront les périodes de misthos et de régime censitaire qui réserve le pouvoir aux plus favorisés. Aristote, Les Politiques, éd. P. Pellegrin, Paris, 1990, VI, 2, 1317b, p. 419 ; IV , 15, 1299b, p. 331.

37. M. Humbert, Les institutions politiques et sociales de l'Antiquité, Paris, 1994

(5 éd.), § 135, p. 90-91.

38. Aristote, Éthique à Nicomaque, éd. J. Voilquin, Paris, 1965, VIII, 10, p. 246-247. 
39. Thomas d'Aquin, Tractatus de rege et regno ad regem Cypri, Opera omnia, t. 27, Opuscula varia, éd. L. Vivès, Paris, 1875, p. 336-412, I, 7, p. 344-346. Ce traité est connu sous le nom du De regno.

40. Thomas d'Aquin, De regno, éd. cit., I, 9, p. 348 : « Magnitudo regie virtutis apparet quod precipue Dei similitudinem gerit, dum hoc agit in regno quod Deus in mundo ».

41. Thomas d'Aquin, De regno, éd. cit., I, 7, p. 344-346

42. Thomas d'Aquin, De regno, éd. cit., I, 8, p. $346:$ «Nam qui divitias habent amplius habere desiderant : qui voluptatibus perfruuntur amplius et perfrui desiderant. Et simile patet in ceteris. [...] Nichil igitur terrenum est quod quietare desiderium possit. Neque igitur terrenum aliquid beatum facere potest, ut possit esse regis conveniens praemium $»$.

43. Thomas d'Aquin, De regno, éd. cit., I, 8, p. 347 : «Solus igitur Deus qui hominis desiderium quietare potest et facere hominem beatum et esse regis conveniens praemium ».

44. Les références bibliques de la récompense sont les versets de Mathieu (Mt 19, 27-30), Luc (Lc 18, 28-30) et Marc (Mc 10, 30).

45. Thomas d'Aquin, De regimine judaeorum, éd. S. E. Frette, t. 27, Paris, 1875, p. 413-416, à la p. 415 .

46. Mt 6, 25-34, $28 ;$ Lc 12,27 ; Lc 10,$7 ; 2$ Th 3, 10.

47. LC 8, 3 .

48. La querelle sur la pauvreté est en partie fondée sur cette question de la propriété et/ou de l'usage de la bourse du Christ. Voir les chapitres que Marsile de Padoue a consacrés à ce sujet dans le Defensor pacis (1328), éd. J. Quillet, Paris, 1968, II, 11-14, p. 276-338.

49. Ne savez-vous pas que les ministres du culte vivent du culte, que ceux qui servent à l'autel partagent avec l'autel ? (1 Co 9, 13). L'Apôtre accepte les dons spontanés (2 Co 11, 7-9 ; Ph 2, 25), qui entraînent des bienfaits pour les donateurs, qu'il remercie en $\mathrm{Ph} 4$, 19, mais il ajoute qu'il faut savoir se contenter du nécessaire $(1 \mathrm{Tm} 6,8)$.

50. Thomas d'Aquin, Somme théologique, Paris, 1984-1986, 4 t., IIa, IIae, q. 87, a. 1, sol. 5, t. 3, p. 550 : « Et ideo Apostolus [1 Co 9, 14-18] noluit uti potestate sibi a domino tradita, ut scilicet acciperet stipendia victus ab his quibus evangelium praedicabat ». 51. Gilles de Rome, De ecclesiastica potestate (1302), éd. R. Schloz, Weimar, 1929, p. 142 : « Decime sunt quasi census possessionum nostrarum, et oblaciones sunt quasi tributa que damus Deo et ecclesie tam pro nobis, quam pro rebus nostris in recognicionem proprie servitutis, quia quedam iura videntur dicere quo ipse decime se habent ut tributa, nihil ad propositum ». Gilles de Rome fait référence à la décrétale De decimis (De decimis, c. 22 Non est in potestate hominum ; c. 23 Ex transissa querela ; c. 26 Tua nobis fraternitas intimavit), X. 3.30.22, 23, 26, Corpus iuris canonici, éd. E. FRIEDBERG, 2 vol., Leipzig, 1879-1882, t. II, 563-565.

52. Thomas d'Aquin, Somme théologique, éd. cit., IIa, IIae, q. 87, a. 1 (" Les dîmes »), t. 4, p. 549 : «Quod enim eis qui divino cultui ministrabant ad salutem populi totius, populus necessaria victus ministraret, ratio naturalis dictat : sicut et his qui communi utilitati invigilant, scilicet principibus et militibus et aliis huiusmodi, stipendia victus debentur a populo »; Ibid., Ia, IIae, q. 105, a. 1, t. 2, p. 701.

53. Thomas d'Aquin, Somme théologique, éd. cit., Ia, IIae, q. 105, a. 1, t. 2, p. 701-703. 54. G. Post, « The Medieval Heritage of a Humanistic Ideal : "Scientia donum Dei est, unde non vendi potest" ", Traditio, 11, 1955, p. 195-234.

55. L'Apparatus de Johannes Teutonicus deviendra la Glossa Ordinaria du Décret. 
56. Thomas d'Aquin, Opera omnia, t. 1, In quattuor libros Sententiarum, Gallarate, 1980, IV , 25, 3, 2b, p. 580. La question posée était : « Utrum sacramenta possint emi et vendi sine simonia?». Thomas a répondu : «Ad octavum, dicendum quod docere artes liberales est actus spiritualis secundo modo ; et dico licet magistris artium vendere labores suos, sed non scientiam sive veritatem quae spiritualis est $»$.

57. Aristote, Éthique à Nicomaque, éd. cit., IX, 1, 1163b 32, p. 263.

58. Thomas d'Aquin, De regno, éd. cit., I, 14, p. 353.

59. Pierre de Tarentaise, Epistola ad Romanos, éd. W. AfFELDT, Die weltliche Gewalt in der Paulus-Exegese. Rm 13, 1-7 in den Römerbriefkommmentarem der lateinischen Kirche bis zum Ende des 13. Jahrhunderts, Gottingen, 1969, p. 307 : « Ipsi enim principes servi pauperum sunt, non domini ».

60. Le terme « office » a alors une application très large puisqu'il désigne toute fonction remplie au service du roi. Cf. A. Rigaudière, Pouvoirs et institutions dans la France médiévale, t. 2, Paris, 1994, p. 273. Le rythme de croissance rapide du nombre des officiers, de 1250 à 1350, se ralentit pendant un siècle pour reprendre après 1450 , J.P. Boudet, « Le bel automne de la culture médiévale ( $x v^{\mathrm{e}}-\mathrm{xv}^{\mathrm{e}}$ siècles), Histoire culturelle de la France, t. 1. Le Moyen Âge, dir. M. Sot, Paris, 1997, p. 225-357, à la p. 251.

61. Nicolas de Lyre, Biblia sacra cum Glossa ordinaria, Anvers, 1617, 6 vol., Dt 17, 16-17, t. II, 1569-1570 : « Non multiplicavit sibi equos, scilicet ad iactatiam, sed solum ad decentiam sui status, et regni defensionem. [...] Neque argenti et auri immensa pondera, id est ad superfluitatem, sed solum ad necessitatem sui status et regni defensionem ».

62. Ph. Buc, L'ambiguïté du Livre. Prince, pouvoir et peuple dans les commentaires de la Bible au Moyen Âge, Paris, 1994, p. 283-286 ; du même, " Pouvoir royal et commentaires de la Bible (1150-1350) », AESC, 44, 1989, p. 691-715.

63. Jean de Salisbury, Policraticus, IV, 6, éd. K. S. B. Keats-Rohan, Turnhout, 1993 (CCCM 118), p. 251 ; voir à ce sujet M. Aurell, L'Empire des Plantagenêt 1154-1224, Paris, 2003, p. 107.

64. Christine de Pizan, Le Livre des faits et bonnes mœurs du roi Charles V le Sage, éd. E. Hicks et Th. Moreau, Paris, 1997 ; Jacques Legrand, Le Livre de bonnes meurs, éd. cit. Evencio Beltran a montré les parentés existant entre les deux auteurs dans l'article : «Christine de Pizan, Jacques Legrand et le Communiloquium de Jean de Galles », Romania, 104, 1983, p. 208-228.

65. JeAn Chrysostome, In epistola ad Romanos, PG 60, Homilia 23, 617 : « Deinde laborem ipsorum ostendes atque miseriam, addit "in hoc ipsum incumbentes" [Rm 13, 6]. Haec enim illorum vita est, hoc studium, ut pace fruaris ». Sur la diffusion de ses écrits, lire J.-P. Bouhot, « Les traductions latines de Jean Chrysostome du v $\mathrm{au}$ $\mathrm{Xv}^{\mathrm{e}}$ siècle ", Traduction et traducteurs au Moyen Âge, Paris, 1989, p. 31-39.

66. Thomas d'Aquin, De regno, éd. cit., I, 7, p. 344.

67. Guillaume Peyraut, De eruditione principum, dans Sancti Thomae Aquinatis. Opera omnia, Parme, 1865, t. 16, p. 390-476, aux p. 246-247.

68. Thomas d'Aquin, Super epistolas S. Pauli lectura, éd. R. Cai, Turin, 1953, t. 1, p. 193. 69. Théodoret (386-458), Interpretatio epistolarum ad Romanos, PG 82, 195 : [Rm 13, 6] «Ideo [...] servientes. Tu enim dormis, et ille communem curam sustinet; et tu domi quidem sedes, ille autem pro pace obtinenda bellum suscipit ».

70. Berthaud de Saint-Denis, Quodlibeta (I, 1-2), éd. J. Leclercq, « Deux questions de Berthaud de Saint-Denys sur l'exemption fiscale du clergé », Études d'histoire du droit canonique dédiées à Gabriel Le Bras, vol. 1, Paris, 1965, p. 611-614, à la p. 614 : « Ideo 
haec publica auctoritas non ligat clericos ad tributa, sed solum ad quorum defensionem et tuitionem principes gratis non tenentur ».

71. L'expression « gens de savoir » fait référence au livre de Jacques Verger, Les gens de savoir en Europe à la fin du Moyen Âge, Paris, 1997.

72. Ce chapitre de l'épître aux Romains a suscité une importante bibliographie sur l'étude des rapports entre pouvoir spirituel et pouvoir temporel à partir de commentaires patristiques et médiévaux. On retiendra principalement les travaux du père jésuite R. Deniel, « "Omnis potestas a Deo". L'origine du pouvoir civil et sa relation à l'Église ", Recherches de Sciences religieuses, 56/1, 1968, p. 43-85, et ceux de W. Affeldt, Die weltliche Gewalt in der Paulus-Exegese. Rm 13, 1-7 in den Römerbriefkommmentarem der lateinischen Kirche bis zum Ende des 13. Jahrhunderts, Gottingen, 1969.

73. Sur les trois « genres » de l'exégèse médiévale (exégèse monastique, exégèse des écoles, exégèse universitaire), lire G. Dahan, L'exégèse chrétienne de la Bible en Occident médiéval, XII ${ }^{\mathrm{e}}$-XIV ${ }^{\mathrm{e}}$ siècle, Paris, 1999, p. 81-120.

74. La traduction de la Bible de Jérusalem est sensiblement différente. Rappelons le texte de la Vulgate : [Rm 13,6] Ideo enim et tributa praestatis ministri enim Dei sunt in hoc ipsum servientes. [7] Reddite omnibus debita cui tributum tributum cui vectigal vectigal cui timorem timorem cui honorem honorem.

75. Jean de la Rochelle, Epistola ad Romanos, Paris, BnF, lat. 15602 , fo $54 \mathrm{v}^{\circ} \mathrm{b}-56 \mathrm{r}^{\circ} \mathrm{a}$, éd. W. Affeldt, op. cit., p. 301-305, p. 304 : « Non ait datis, sed praestatis, quasi scilicet reddituris $»$.

76. J.-P. Torrell, Initiation à saint Thomas d'Aquin. Sa personne et son œuvre, Vestigia 13, Paris-Fribourg, 1993, p. 365-371.

77. Thomas d'Aquin, Super epistolas S. Pauli lectura, éd. cit., p. 192-193 : « Et ideo cum principes nostri suo regimine Deo ministrent, a populo debent tributa suscipere, quasi stipendia sui ministerii, non autem ita quod hoc debeant sibi computare pro praemio [...]. Sed attendendum est : cum tributa dicat regibus esse debita quasi laboris stipendium, dupliciter peccare possunt principes accipiendo tributa ». J.-E. Stroobant de Saint-Éloy traduit stipendium sui ministerii ou stipendium laboris par «salaire de leur ministère » et « le salaire du travail », Commentaire de l'épître aux Romains, trad. J.-E. Stroobant de Saint-Éloy, Paris, 1999, p. 1037 et 1041. Je préfère conserver la connotation militaire du mot « solde » qui rend mieux le sens d'entretien que contient le mot stipendium.

78. Pierre de Tarentaise, Epistola ad Romanos, éd. cit., p. 307 : «Item super illud : in hoc ipsum servientes ; glossa : id est propter tributum ; contra : ergo praemium principis est temporale. Respondeo : tributum non est praemium laboris, sed stipendium laborantis ».

79. Pierre de Tarentaise oppose labor à laborans et praemium à stipendium, distinguant la récompense céleste (Béatitude) et la récompense terrestre (tributs). 80. Henri de Gand, Opera omnia, éd. R. Macken, 5 vol., Louvain, 1978-1985, t. 13, Quodlibeta, IX, 31, p. 327-339, à la p. 328 : « Ubi dicit Glossa super illud "praestatis" : Ad ostendum subiectionem ; quasi dicat : "Non redditis", quia reddent solvendo, dum in defensione pugnant pro patria ». Le théologien démarque la glose qui, la première, avait comparé les sens des verbes solvere, praestare, reddere.

81. Richard de Mediavilla, Quodlibeta, III, 27, Paris, BnF, lat. 14305, fo $194 \mathrm{v}^{\circ} \mathrm{b}-195 \mathrm{r}^{\circ} \mathrm{b}$. 
82. M. Mauss, « Essai sur le don. Forme et raison de l'échange dans les sociétés archaïques ", L'Année anthropologique, 1923-1924, repris dans Sociologie et anthropologie, Paris, 1973, p. 145-279.

\section{RÉSUMÉS}

Le Livre de bonnes meurs a été offert en 1410 au duc de Berry par le religieux augustin Jacques Legrand. Vers 1490, un artiste de la vallée de la Loire a orné une copie de ce traité moral de 53 miniatures. L'une d'entre elles est énigmatique : représente-t-elle « le roi levant l'impôt-or sur

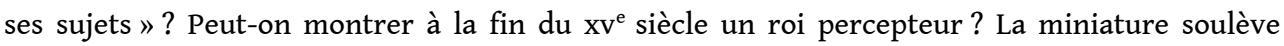
également la question alors posée par des théologiens, Mendiants pour la plupart, sur la nature du prélèvement fiscal : l'impôt est-il « le salaire du roi »? Certains esprits subtils vont distinguer - comme cela avait été fait pour justifier la rémunération des maîtres ou des prédicateurs - ce qui relevait de la fonction ministérielle du prince, gratuite, de la pratique pénible du gouvernement, à laquelle il n'était pas tenu gratuitement.

"The King refuses the gold of his subjects». Study of a miniature of Jacques Legrand's Livre de bonnes meurs (ca 1410). The Livre de bonnes meurs was dedicated in 1410 by the Augustinian friar Jacques Legrand to the Duke of Berry. Towards the end of the century, an anonymous artist decorated a copy of this moral treatise with 53 miniatures. One of them is enigmatic: is it about the King levying gold taxes from his subjects? Is it possible to represent the King as a tax collector at the end of the 15th Century? The miniature also raises the question discussed at that time by the theologians - mostly Mendicants - about tax collecting : is taxation the "King's wage" ? Some subtle minds answered by taking up the distinction already alleged to justify the remuneration of teachers and preachers : on one hand, the ministerial function of the monarch is free of charge, and on the other hand, the government includes a laborious practical dimension, which is not a king's free duty.

\section{INDEX}

Mots-clés : Jacques Legrand († 1415), miniature, impôt, salaire, exégèse

Keywords : taxation, remuneration, exegesis

\section{AUTEUR}

\section{LYDWINE SCORDIA}

Département d'Histoire, Faculté des lettres et sciences humaines, Université de Rouen, F-76821 Mont-Saint-Aignan Cedex. 